\title{
Effects of glucagon-like peptide-1(7-36) amide on neurohypophysial and cardiovascular functions under hypo- or normotensive hypovolaemia in the rat
}

\author{
E Bojanowska and B Stempniak \\ Department of Pathophysiology, Medical University of Lodz, 60 Narutowicza St., Pl-90-136 Lodz, Poland \\ (Requests for offprints should be addressed to E Bojanowska)
}

\begin{abstract}
To date, glucagon-like peptide 1(7-36) amide (tGLP-1) has been found to affect the neurohypophysial and cardiovascular functions in normotensive and normovolaemic rats. The aim of the present study was to investigate possible effects of tGLP-1 on the mean arterial blood pressure and the release of vasopressin and oxytocin under conditions of blood volume depletion in the rat. In the first series of experiments, the animals were injected i.p. with either $0 \cdot 15 \mathrm{M}$ saline or $30 \%$ polyethylene glycol (PEG). PEG caused an 18\% reduction of blood volume $1 \mathrm{~h}$ after injection. No significant changes in the mean arterial blood pressure were found in either normo- or hypovolaemic rats during the experiment. tGLP-1 injected i.c.v. at a dose of $1 \mu \mathrm{g} / 5 \mu \mathrm{l} 1 \mathrm{~h}$ after the i.p. injection increased similarly the arterial blood pressure in normoand hypovolaemic rats. The plasma vasopressin/oxytocin
\end{abstract}

concentrations were markedly elevated in hypovolaemic animals and tGLP-1 further augmented the release of both hormones. In the second study, hypovolaemia was induced by double blood withdrawal. The haemorrhage resulted in a marked decrease of the mean arterial blood pressure and in the elevated plasma vasopressin/oxytocin concentrations. tGLP-1 injected immediately after the second blood withdrawal increased the arterial blood pressure. In parallel, tGLP-1 enhanced significantly vasopressin and oxytocin secretion when compared with haemorrhaged, saline-injected rats. The results of this study indicate that tGLP-1 may affect the arterial blood pressure and the secretion of neurohypophysial hormones under pathological conditions brought about by blood volume depletion.

Journal of Endocrinology (2002) 172, 303-310

\section{Introduction}

Hypovolaemia and hypotension are known to be powerful stimuli increasing the release of arginine vasopressin (AVP) and oxytocin (OXY) in the rat (Schiltz et al. 1997, Kadekaro et al. 1998). The reduction of blood volume is mainly detected by cardiac stretch (volume) receptors whilst the reduction of blood pressure affects the activity of arterial baroreceptors (Renaud 1996, Share 1996, Thrasher \& Keil 2000). The information is then transmitted to the nucleus of the solitary tract (NST) and to other, yet not fully recognised, structures involved in the control of the hypothalamo-neurohypophysial complex (Smith et al. 1995, Renaud 1996, Share 1996). As a result, the electrophysiological activity of vasopressinergic and oxytocinergic neurons changes in a manner characteristic for each of these neuronal systems (Poulain \& Wakerley 1982).

The new pattern of activity depends on the altered synaptic transmission within the hypothalamoneurohypophysial complex. Numerous neurotransmitters and neuromodulators have been shown to influence the function of magnocellular hypothalamic neurons under conditions of hypovolaemia (Yokoi et al. 1996, Kadekaro et al. 1998, Ueta et al. 1998, Yamaguchi et al. 1998). In this respect, it is of interest to investigate possible effects of another brain peptide, glucagon-like peptide 1(7-36) amide (tGLP-1), postulated to be a neuromodulator of the autonomic nervous system, on the hypovolaemia-induced release of neurohypophysial hormones.

tGLP-1 was found in cell bodies localised in some brain structures including the NST as well as in nerve fibres projecting to the paraventricular nuclei (PVN) and supraoptic nuclei (SON) (Jin et al. 1988). Within nerve terminals, tGLP-1 was detected in the synaptosome fraction, thus implying its role as a neurotransmitter or neuromodulator (Kreymann et al. 1989). Moreover, tGLP-1 binding sites or mRNA for tGLP-1 receptor were found to be widely distributed in the rat brain. For example, the hypothalamic magnocellular neurons express mRNA for tGLP-1 receptor (Navarro et al. 1996, Shughrue et al. 1996, Merchenthaler et al. 1999, Zueco et al. 1999). Specific tGLP-1 binding sites were also demonstrated in the rat neurohypophysis (Göke et al. 
1995, Satoh et al. 2000). I.c.v. injected tGLP-1 was shown to stimulate the basal release of neurohypophysial hormones in normotensive rats (Larsen et al. 1997, Bojanowska \& Stempniak 2000). What is more, centrally injected tGLP-1 was also shown to affect the function of the cardiovascular system in the rat (Barragán et al. 1999, Bojanowska \& Stempniak 2000).

Therefore, the aim of the present study was to investigate possible effects of tGLP-1 on the release of AVP and $\mathrm{OXY}$ as well as blood pressure under hypotensive and non-hypotensive hypovolaemia.

\section{Materials and Methods}

\section{Animals}

The experiments were carried out on male Wistar rats (250-350 g). They were kept on a $14 \mathrm{~h}$ light:10 h darkness cycle. Food and water were freely available.

\section{Experimental procedure}

Urethane-anaesthetised (1.2 g/kg body weight (BW), i.p. injection) rats were fitted with heparinised polyethylene cannulae (outer diameter $0.80 \mathrm{~mm}$, inner diameter $0.50 \mathrm{~mm}$; Critchley Electrical Ltd, Silverwater B.C., Australia) inserted in both femoral arteries and the femoral vein as well as with an i.c.v. cannula implanted in the lateral brain ventricle. One arterial cannula was connected to a BP-1 blood pressure monitor via a BLPR transducer (World Precision Instruments, Berlin, Germany) to monitor continuously the blood pressure.

Each experiment started after an equilibration period, when blood pressure was found to be stable. Then the animals under sustained urethane anaesthesia were subjected to the experimental procedure which resulted finally in non-hypotensive or hypotensive hypovolaemia (see below).

Series 1: effects of tGLP-1 on the hormonal and cardiovascular response to non-hypotensive hypovolaemia Non-hypotensive hypovolaemia was induced by polyethylene glycol (PEG) treatment. Systemically injected PEG was proved to reduce the blood volume without affecting significantly the arterial blood pressure and the plasma osmolality. Moreover, the release of both neurohypophysial hormones was shown to be enhanced markedly under PEG-induced hypovolaemia (Stricker \& Verbalis 1986, Forsling et al. 1991).

At the beginning of the experiment, a $1 \mathrm{ml}$ blood sample was taken from the femoral artery. This sample was immediately replaced with an equal volume of sterile isotonic saline infused into the femoral vein. Twenty minutes later, 30\% (w/v) PEG (molecular mass $3350 \mathrm{Da}$; Sigma Aldrich, Poznan, Poland) dissolved in $0.15 \mathrm{M} \mathrm{NaCl}$ or saline alone (in control animals) was injected i.p. in a dose of $20 \mathrm{ml} / \mathrm{kg} \mathrm{BW}$. The blood pressure was recorded every $10 \mathrm{~min}$ over a $70 \mathrm{~min}$ period after PEG injection. One hour after PEG injection, $1 \mu \mathrm{g}$ tGLP-1 (Calbiochem, Unimarket, Poznan, Poland) dissolved in $5 \mu \mathrm{l}$ isotonic saline or the vehicle alone was infused i.c.v. over $1 \mathrm{~min}$. The second blood sample was taken $10 \mathrm{~min}$ later. The tGLP-1 dose and the time of sampling were selected on the basis of previous experiments (Bojanowska \& Stempniak 2000). The second sample was replaced with an equal volume of saline containing cells from the first sample. Further analyses were performed as described below.

Series 2: effects of tGLP-1 on the hormonal and cardiovascular response to hypotensive hypovolaemia Hypotensive hypovolaemia was induced by double blood withdrawal. Two successive blood samples of $10 \mathrm{ml} / \mathrm{kg} \mathrm{BW}$ each were taken from the femoral artery; the second sample was obtained $10 \mathrm{~min}$ after the first. Then $1 \mu \mathrm{g}$ tGLP-1, dissolved in $5 \mu \mathrm{l}$ isotonic saline, or vehicle alone was injected i.c.v. The third blood sample of $1 \mathrm{ml}$ was taken $10 \mathrm{~min}$ later. This sample, however, was immediately replaced with the appropriate volume of saline containing blood cells from the previous sample. The control, i.e. non-haemorrhaged rats, were subjected to similar procedures except that blood samples were immediately replaced with the same volume of saline containing resuspended blood cells; the first sample was replaced with saline only. The mean arterial blood pressure was recorded every $5 \mathrm{~min}$ throughout the experiment. At the end of each experiment, further analyses were performed as described below.

\section{Analyses}

Blood samples obtained during experiments were immediately centrifuged at 3000 r.p.m. $(550 \mathrm{~g}$ ) for $3 \mathrm{~min}$ at $4{ }^{\circ} \mathrm{C}$; the plasma was separated and kept at $-20{ }^{\circ} \mathrm{C}$ until assayed. The blood cells were resuspended in the appropriate volume of isotonic saline and, when necessary, reinjected to the animal. Haematocrit and plasma osmolality were also determined in all blood samples. The latter analysis was performed by freezing-point depression by means of a semimicro-osmometer (Knauer GmBH, Berlin, Germany).

At the end of each experiment the position of the i.c.v. cannula was verified by injection of $5 \mu 15 \%$ (w/v) Evans blue solution into the ventricular system. Simultaneously, the blood volume was determined in all animals. Each rat was injected i.v. with $50 \mu \mathrm{l}$ 5\% Evans blue solution. The animals were decapitated $10 \mathrm{~min}$ later. The trunk blood was collected into heparinised tubes, centrifuged, and the blood plasma used for analysis. The plasma concentrations of the dye were estimated using a SPEKOL spectrophotometer (Carl Zeiss, Jena, Germany) at $625 \mathrm{~nm}$. After 


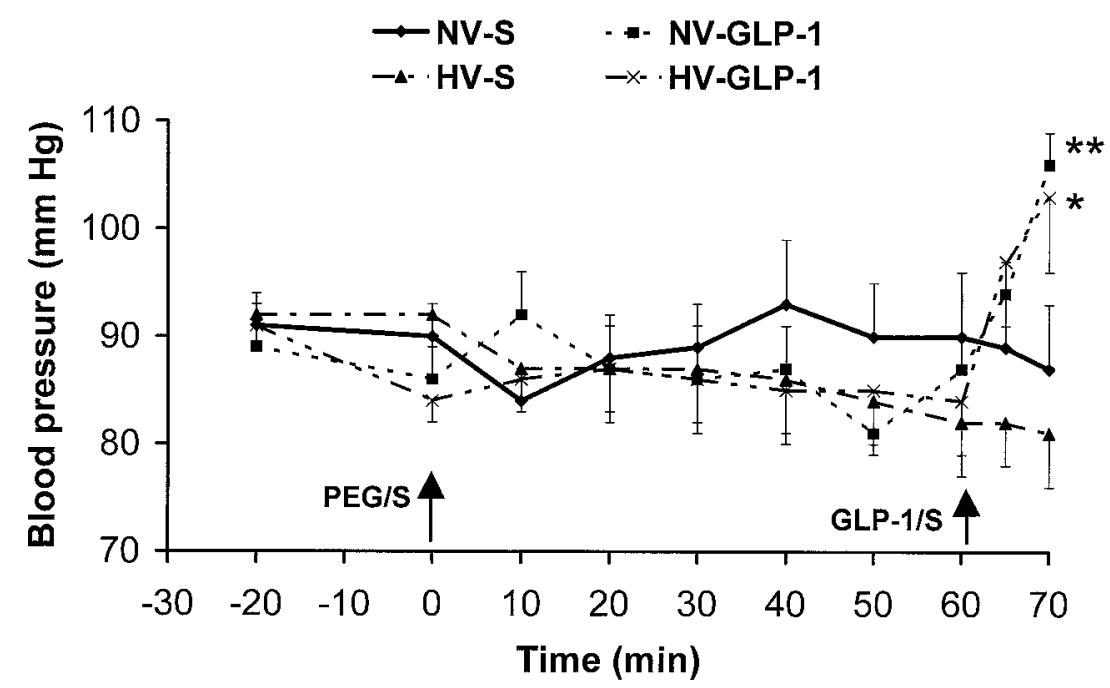

Figure 1 Effects of glucagon-like peptide 1 (7-36) amide (tGLP-1) on the mean arterial blood pressure in normovolaemic (NV) rats or hypovolaemic (HV), PEG-treated rats. The animals were injected i.c.v. with either saline (S) or $1 \mu \mathrm{g}$ tGLP-1. ANOVA showed the time $\times$ drug treatment interaction to be significant $(P<0 \cdot 001)$. Further comparisons were made in relation to the initial value in each group $(n=8) .{ }^{*} P<0 \cdot 05,{ }^{*} P<0 \cdot 002$.

decapitation, the neurohypophysis was isolated and homogenised in $0.5 \%(\mathrm{v} / \mathrm{v})$ acetic acid.

\section{$R I A$}

AVP and OXY were extracted from plasma using C18 Sep-Pak Plus cartridges (Waters Co., Milford, MA, USA). The recoveries of synthetic AVP and OXY added to plasma samples during extraction were $70 \%$; the hormone levels were not corrected for recovery. The hormone concentrations were measured as described previously (Lewandowska et al. 1995, Bojanowska et al. 1999). OXY and AVP antisera were raised by Dr M Orlowska-Majdak (Department of Physiology, Medical University of Lodz, Poland). Cross-reactivity with AVP for OXY antibodies was $1.1 \%$ and cross-reaction with OXY for AVP antibodies was less than $1 \%$. The intra-assay coefficients of variation for AVP and OXY assay were 4 and 5.5\% respectively. In each series of experiments, samples were tested in a single assay to avoid interassay variability.

\section{Statistical analysis}

Results are expressed as means \pm S.E.M. of six to eight animals. The effects of tGLP-1 on the plasma hormone level, mean arterial blood pressure, plasma osmolality, and haematocrit in normo- or hypovolaemic rats over the time course of the experiment were estimated using three-way ANOVA followed by the post-hoc least significant difference (LSD) test. The neurohypophysial response to tGLP-1 and PEG or haemorrhage was analysed using two-way ANOVA and an LSD test for multiple comparisons (Statistica, StatSoft, Krakow, Poland) with $P<0 \cdot 05$ considered to be significant.

\section{Results}

\section{Series 1: $t G L P-1$ and PEG-induced hypovolaemia}

The PEG treatment reduced the blood volume by $18 \%$. No significant differences between experimental groups were found as to the plasma osmolality within a $70 \mathrm{~min}$ period after i.p. saline or PEG injection.

The effect of tGLP-1 on the arterial blood pressure in normo- and hypotensive rats is shown in Fig. 1. The reduction of blood volume did not affect significantly blood pressure $1 \mathrm{~h}$ after PEG injection. tGLP-1 increased markedly the mean arterial blood pressure in both normoand hypovolaemic rats as compared with the ' 0 ' time in each group. The blood pressure measured in normovolaemic, tGLP-1-treated rats at the 70th $\min$ of the experiment (i.e. $10 \mathrm{~min}$ after i.c.v. injection) was also significantly higher than the value obtained in the normovolaemic, saline-injected group $(P<0.003)$ at the same time. Similar results were obtained when the comparisons were made between PEG-treated animals injected with tGLP-1 or saline $(P<0 \cdot 001)$. On the other hand, blood pressure after tGLP-1 injection did not differ significantly in normo- and hypovolaemic animals.

Figure 2 shows the effects of tGLP-1 on the release of neurohypophysial hormones under normotensive 

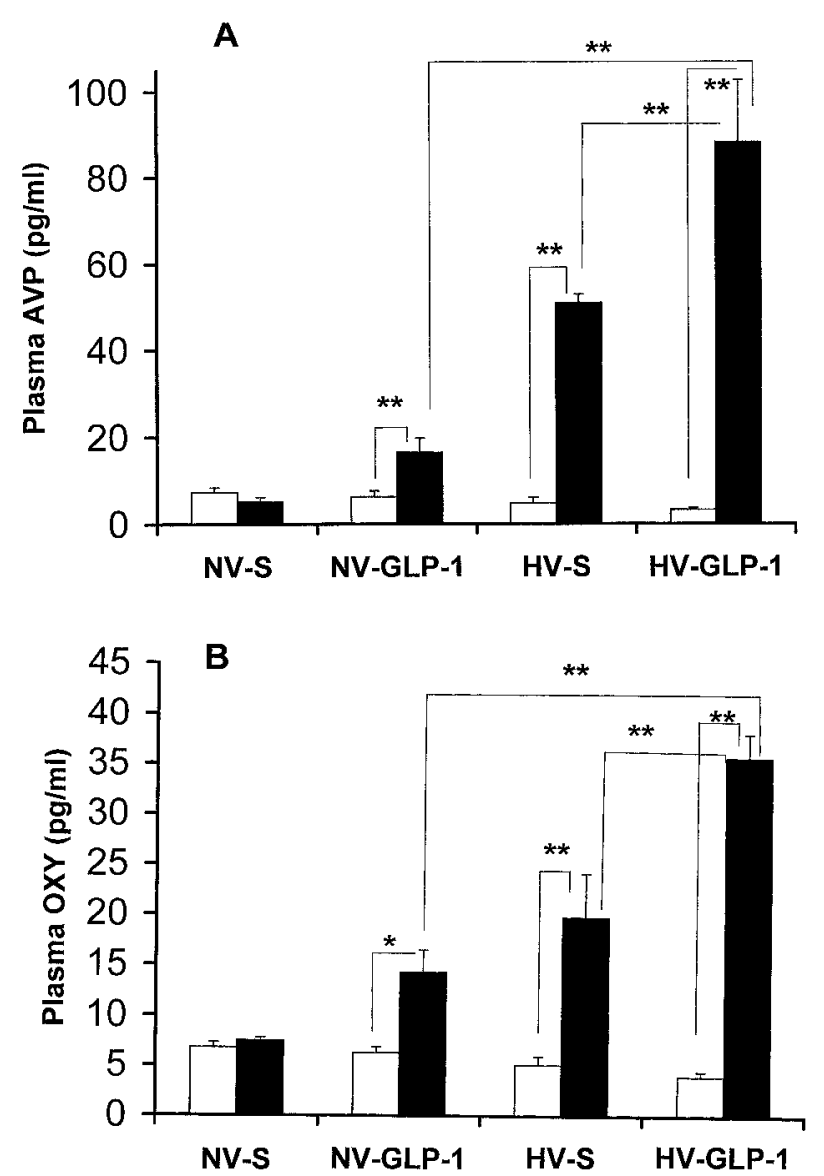

Figure 2 Effects of tGLP-1 on the plasma arginine vasopressin (AVP) and oxytocin (OXY) concentrations in NV rats or HV, PEG-treated rats injected i.c.v. with either saline (S) or $1 \mu \mathrm{g}$ tGLP-1. Open bars represent the initial hormone level (the ' 0 ' time), filled bars the final hormone concentration (at the 70th min, i.e. $10 \mathrm{~min}$ after the i.c.v. injection) ( $n=7-8)$. ANOVA revealed significant time-, volume- and treatment-dependent effects (all $P<0 \cdot 001$ ) on the plasma AVP/OXY concentrations. The interactions time $\times$ volume and time $\times$ drug treatment for OXY level as well as all interaction terms for AVP level were also found to be significant (all $P<0 \cdot 01$ ). ${ }^{*} P<0 \cdot 02,{ }^{*} P<0 \cdot 001$.

hypovolaemia. PEG-induced hypovolaemia markedly enhanced the release of AVP in saline-injected rats. tGLP-1 significantly increased the plasma AVP level in normovolaemic animals when compared with the initial values in this group as well as with the hormone concentration in saline-injected, normovolaemic controls $(P<0 \cdot 001)$. On the other hand, the plasma hormone concentration in hypovolaemic tGLP-1-treated rats was markedly higher than in hypovolaemic, saline-injected animals at the end of the experiment (Fig. 2A). tGLP-1 enhanced markedly $(P<0 \cdot 02)$ the OXY release in normovolaemic rats and increased significantly $(P<0 \cdot 001)$ the hormone secretion previously enhanced by the reduction of blood volume (Fig. 2B). $\square$ AVP $\square O X Y$

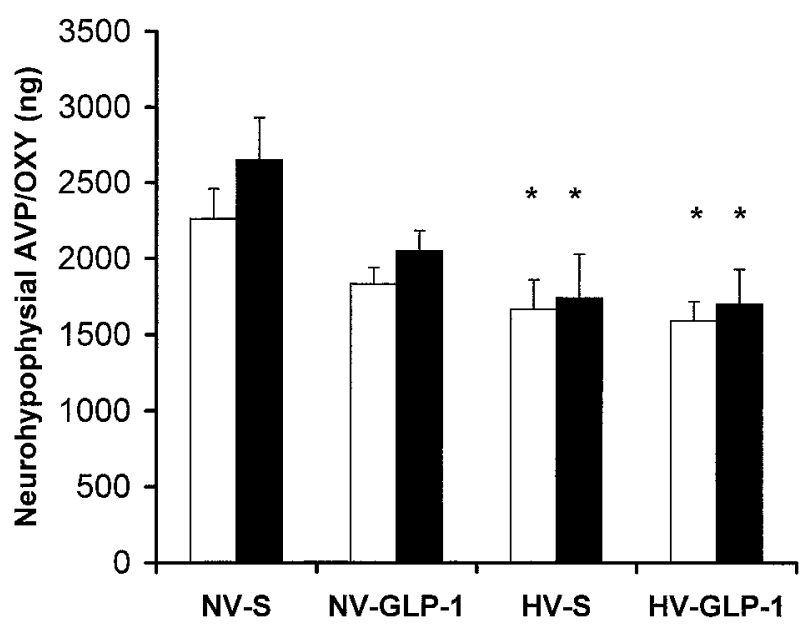

Figure 3 Neurohypophysial AVP and OXY content in NV rats or HV, PEG-treated rats injected i.c.v. with saline (S) or $1 \mu \mathrm{g}$ tGLP-1. ANOVA indicated that the neurohypophysial AVP/OXY content was affected markedly by hypovolaemia $(P<0 \cdot 02$ and $P<0 \cdot 01$ for AVP and OXY respectively) but not by drug treatment. Further comparisons were made in relation to normovolaemic salineinjected (NV-S) rats $(n=6-8) .{ }^{*} P<0 \cdot 01$.

As shown in Fig. 3, the AVP and OXY content in the neurohypophysis was significantly decreased in PEGtreated rats when compared with normovolaemic controls. tGLP-1 did not further modify the neurohypophysial hormone storage.

\section{Series 2: tGLP-1 and haemorrhage-induced hypovolaemia}

The effects of blood withdrawal on plasma osmolality, haematocrit and blood volume are shown in Table 1 . The double bleeding caused $22 \%$ reduction of the blood volume as compared with non-haemorrhaged controls. The plasma osmolality in both normo- and hypovolaemic rats did not change significantly during the experiment. On the other hand, ANOVA showed a significant $(P<0 \cdot 01)$ effect of the time course $\times$ blood volume interactions on the haematocrit index. Haematocrit measured in the last blood sample in non-haemorrhaged rats did not differ significantly from the initial value. In haemorrhaged animals, however, haematocrit decreased markedly after bleeding (as compared with the initial value) in both saline- and tGLP-1-injected rats in both groups.

The effects of tGLP-1 on the arterial blood pressure in haemorrhaged animals are shown in Fig. 4. The blood pressure in haemorrhaged rats injected i.c.v. with saline decreased significantly after bleeding whilst blood pressure was stable in the respective non-haemorrhaged controls during the experiment. When, however, tGLP-1 was injected immediately after the second blood withdrawal, blood pressure tended to rise over a $10 \mathrm{~min}$ period after 
Table 1 Plasma osmolality, haematocrit and blood volume (means \pm S.E.M., number of animals in parentheses) in haemorrhaged (two subsequent blood withdrawals of $10 \mathrm{ml} / \mathrm{kg}$ BW each) and non-haemorrhaged rats injected i.c.v. with glucagon-like peptide 1 (7-36) amide tGLP-1. Plasma osmolality was measured before blood withdrawal (1), $10 \mathrm{~min}$ after the first haemorrhage (2), and $10 \mathrm{~min}$ after the second haemorrhage (3). Haematocrit was measured in the initial and final blood sample. The mean blood volume was averaged from samples obtained from all (i.e. saline- or tGLP-1-injected) non-haemorrhaged and haemorrhaged rats

\begin{tabular}{|c|c|c|c|c|c|c|}
\hline & \multicolumn{3}{|c|}{ Plasma osmolality (mOsm/kg $\left.\mathrm{H}_{2} \mathrm{O}\right)$} & \multicolumn{2}{|c|}{ Haematocrit (\%) } & \multirow[b]{2}{*}{ Blood volume $(\mathrm{ml})$} \\
\hline & 1 & 2 & 3 & 1 & 3 & \\
\hline \multirow{3}{*}{$\begin{array}{l}\text { Group } \\
\text { Non-haemorrhaged, saline-injected } \\
\text { Non-haemorrhaged, tGLP-1-injected }\end{array}$} & & & & & & \\
\hline & $287 \pm 4(7)$ & $280 \pm 2(7)$ & $284 \pm 7(6)$ & $48 \pm 1(7)$ & $47 \pm 1(7)$ & \multirow{2}{*}{$36 \pm 2(14)$} \\
\hline & $289 \pm 4(6)$ & $293 \pm 6(6)$ & $293 \pm 5(6)$ & $48 \pm 2(7)$ & $48 \pm 1(7)$ & \\
\hline $\begin{array}{l}\text { Haemorrhaged, saline-injected } \\
\text { Haemorrhaged, tGLP-1-injected }\end{array}$ & $\begin{array}{l}280 \pm 4(8) \\
281 \pm 2(8)\end{array}$ & $\begin{array}{l}289 \pm 4(8) \\
288 \pm 5(8)\end{array}$ & $\begin{array}{l}285 \pm 3(8) \\
288 \pm 5(8)\end{array}$ & $\begin{array}{l}49 \pm 1(8) \\
49 \pm 1(8)\end{array}$ & $\begin{array}{l}43 \pm 1(8)^{\mathrm{a}} \\
45 \pm 1(8)^{\mathrm{b}}\end{array}$ & $28 \pm 2(16)$ \\
\hline
\end{tabular}

${ }^{\mathrm{a}} \mathrm{P}<0 \cdot 001,{ }^{\mathrm{b}} \mathrm{P}<0 \cdot 01$ (as compared with the initial value in each group).

drug injection. On the other hand, blood pressure in non-haemorrhaged rats injected with tGLP-1 was markedly $(P<0 \cdot 02)$ higher than the control value in saline-injected rats $10 \mathrm{~min}$ after the i.c.v. injection as well as than the initial value in the same group (normovolaemic, tGLP-1-treated rats).

Figure 5 shows the effects of tGLP-1 on neurohypophysial hormone release under hypotensive hypovolaemia. Both the AVP and OXY release were significantly augmented in haemorrhaged rats $10 \mathrm{~min}$ after the second bleeding. tGLP-1 injected after the second blood withdrawal markedly increased the plasma level of both hormones as compared with the initial values in this group and the respective values in hypovolaemic controls at the same time ( $P<0 \cdot 001$ and $P<0 \cdot 01$ for AVP and OXY respectively). In normovolaemic rats, tGLP-1 also increased the plasma AVP/OXY concentrations, the respective values being, however, significantly $(P<0.001)$ lower than the hormone level in haemorrhaged, tGLP-1-injected rats.

The neurohypophysial levels of AVP and OXY are summarised in Table 2. The neurohypophysial AVP/ OXY storage was not affected markedly by either blood volume depletion or drug treatment. In particular, the neurohypophysial hormone content in all groups did not differ significantly from the control values in non-haemorrhaged, saline-injected rats.

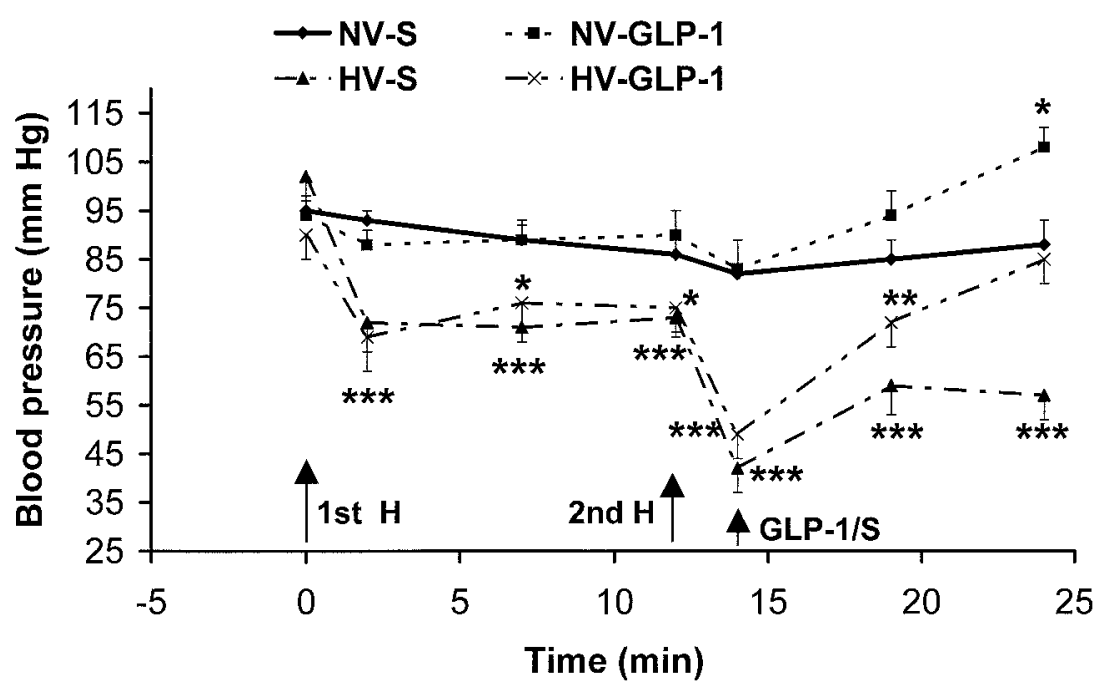

Figure 4 Effects of tGLP-1 on the mean arterial blood pressure in NV or HV rats. Hypovolaemia was produced by double blood withdrawal. The arrows indicate the $1 \mathrm{st}$ and 2nd haemorrhage $(\mathrm{H})$ as well as the i.c.v. saline $(\mathrm{S})$ or tGLP-1 injection. ANOVA revealed significant effects of the volaemic status $(P<0.001)$, time $(P<0 \cdot 001)$, and drug treatment $(P<0 \cdot 02)$ on the mean arterial blood pressure. Moreover, some interaction terms were also found to be significant (volaemia $\times$ time, $P<0.001$ and time $\times$ treatment, $P<0 \cdot 001$ ). Further comparisons were made in relation to the initial value in each group $(n=7-8) .{ }^{*} P<0 \cdot 05$, ${ }^{*} P<0 \cdot 01,{ }^{* * *} P<0 \cdot 001$. 


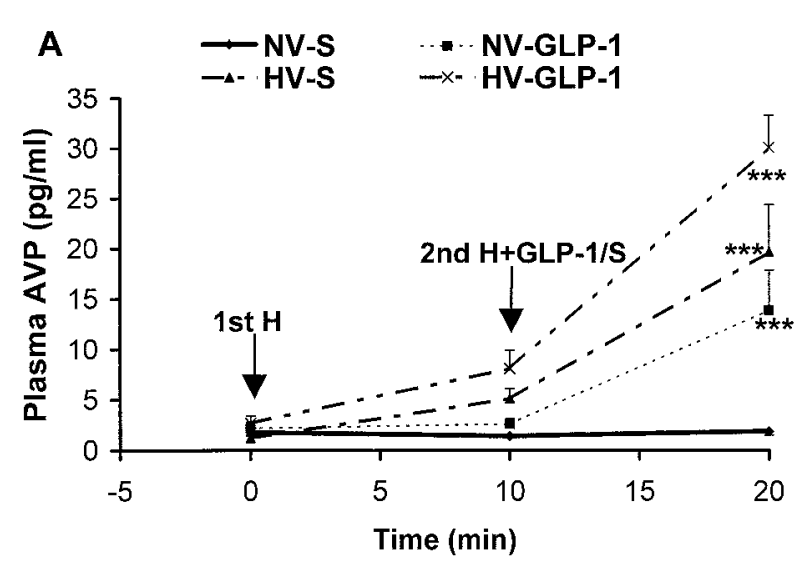

B
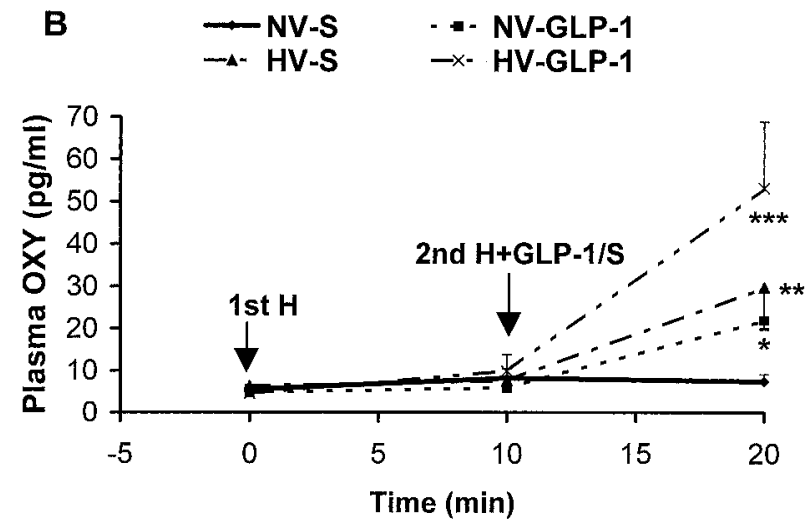

Figure 5 Effects of tGLP-1 on the plasma AVP and OXY concentrations in NV or HV rats injected i.c.v. with either saline (S) or $1 \mu \mathrm{g}$ tGLP-1. Hypovolaemia was produced by double blood withdrawal. The arrows indicate the $1 \mathrm{st}$ and 2 nd haemorrhage $(\mathrm{H})$ as well as the i.c.v. saline $(S)$ or tGLP-1 injection. ANOVA showed significant time- $(P<0 \cdot 001)$, volume- $(P<0.001)$ and treatment-dependent $(P<0 \cdot 001)$ effects on the plasma AVP concentrations. Moreover, the interactions time $\times$ treatment and time $\times$ volume were shown to be significant $(P<0 \cdot 01$ and $P<0.001$ respectively). With regard to the plasma OXY level, ANOVA revealed significant time- $(P<0 \cdot 001)$, volume- $(P<0 \cdot 01)$, and drug-dependent $(P<0 \cdot 05)$ as well as interaction-dependent (time $\times$ volume, $P<0 \cdot 01$ and time $\times$ treatment, $P<0 \cdot 02$ ) effects. Further comparisons were made in relation to the initial value in each group $(n=7-8) .{ }^{*} P<0 \cdot 05,{ }^{*} * P<0 \cdot 01,{ }^{* *} P<0 \cdot 001$.

\section{Discussion}

The results of the present study indicate that tGLP-1 may affect blood pressure and neurohypophysial hormone release under pathological conditions brought about by blood volume depletion in the rat.

In order to produce hypovolaemia, two experimental procedures were employed in this study. The PEG injection reduced the blood volume without any marked changes in the arterial blood pressure. On the contrary, in the second series of experiments, two subsequent blood withdrawals resulted not only in diminished blood volume but also in decreased arterial blood pressure. The degree of the blood volume reduction was comparable in both series of experiments (i.e. 20\%).

\section{Series 1: tGLP-1 and PEG-induced hypovolaemia}

The PEG-induced hypovolaemia is rather moderate and develops gradually in time (Stricker \& Verbalis 1986). It seems that, under these conditions, the compensatory mechanisms are able to maintain blood pressure at a relatively stable level in spite of the disturbed volaemic status. This could account for a similar cardiovascular response to tGLP-1 occurring in both normo- and hypovolaemic rats. On the other hand, the release of AVP and OXY was markedly increased under normotensive hypovolaemia and tGLP-1 additionally enhanced the secretion of both hormones in hypovolaemic rats. The latter is a key observation in our study since, to date, tGLP-1 has been found to modulate the secretion of neurohypophysial hormones under basal conditions only (Larsen et al. 1997, Bojanowska \& Stempniak 2000). It is also noteworthy that the hormonal response to tGLP-1 in PEG-treated animals was markedly augmented when compared with the hormone secretion in tGLP-1-injected normovolaemic rats. This indicates that the stimulatory effects of hypovolaemia and tGLP-1 on the neurohypophysial hormone release are additive. Hence, these findings suggest that tGLP-1 seems to be a relatively powerful stimulus for vasopressinergic and oxytocinergic neurons under conditions of hypovolaemia.

Table 2 Neurohypophysial arginine vasopressin (AVP) and oxytocin (OXY) content (means \pm S.E.M., number of animals in parentheses) in haemorrhaged (two subsequent blood withdrawals of $10 \mathrm{ml} / \mathrm{kg}$ BW each) and non-haemorrhaged rats injected i.c.v. with tGLP-1

\section{Group}

Non-haemorrhaged, saline-injected Non-haemorrhaged, tGLP-1-injected

Haemorrhaged, saline-injected Haemorrhaged, tGLP-1-injected

\section{AVP (ng)}

$1736 \pm 210(7)$

$1608 \pm 175(8)$

$1501 \pm 336(7)$

$1537 \pm 126(8)$
OXY (ng)

$1763 \pm 108(6)$

$1684 \pm 190(6)$

$1497 \pm 188(7)$

$1725 \pm 129(7)$ 
On the contrary, the neurohypophysial AVP/OXY content was decreased in hypovolaemic rats; tGLP-1 did not alter the hormone storage regardless of the volaemic status of the animals. Possibly, the bolus injection of tGLP-1 produced only a short-term hormone output, which was too weak to cause significant changes in the neurohypophysial AVP/OXY content. A previous report (Bojanowska \& Stempniak 2000) showed that the effect of tGLP-1 on the AVP/OXY release is transient and disappears as soon as $15 \mathrm{~min}$ after the injection. On the other hand, the gradual blood volume reduction may lead to the noticeable depletion of neurohypophysial hormone stores as found in this study.

\section{Series 2: tGLP-1 and haemorrhage-induced hypovolaemia}

The double blood withdrawal resulted in a marked fall in the mean arterial blood pressure accompanied by an elevated AVP/OXY level in the blood plasma. In contrast, the neurohypophysial hormone content was not altered by either bleeding or the bolus tGLP-1 injection. Apparently, the release of both hormones was not enhanced enough to affect significantly the AVP/OXY stores in the neurohypophysis during a relatively short time after the blood withdrawal or the peptide injection.

In control (saline-injected) rats, haemorrhage caused an acute hypotensive response followed by a moderate rise of the arterial blood pressure after the blood withdrawal. It seems that the initial decrease in blood pressure is a result of either suppressed sympathetic or enhanced parasympathetic activity (Ullman 2000). tGLP-1 not only accelerated the compensatory response immediately after the second bleeding but also increased blood pressure to the level comparable with the initial value. Because AVP per se is known to be a powerful vasoconstrictor, the enhanced hormone secretion, additionally induced by tGLP-1, could also be considered as an important compensatory mechanism involved in the rise of blood pressure in haemorrhaged animals (Szczepańska-Sadowska 1996). Interestingly, blood pressure in haemorrhaged, salineinjected rats decreased markedly in spite of the enhanced vasopressor response. This suggests a primary role of tGLP-1 for regulation of blood pressure in blood-depleted rats under conditions of this experiment. An earlier report (Barragán et al. 1999) showed that the vagus nerve mediates the effect of centrally injected tGLP-1 on the arterial blood pressure. Moreover, the vagal tone is known to be altered under hypovolaemia. In the present study, however, tGLP-1 was found to produce similar, stimulatory effects as to blood pressure in normo- and hypotensive rats regardless of whether hypovolaemia was gradual or acute. Therefore, we hypothesise that tGLP-1 can influence equally both the basal and altered parasympathetic activity in terms of the control of arterial blood pressure. This effect is likely to be mediated by the NST and/or the area postrema, both of them being known not only to be involved in the control of blood pressure under haemorrhage (Badoer et al. 1992) but also to contain mRNA for tGLP-1 receptor (Merchenthaler et al. 1999). I.c.v. injected tGLP-1 was also demonstrated to activate selectively neurons of these structures (Van Dijk et al. 1996).

Moreover, tGLP-1 increased the AVP/OXY release previously augmented by haemorrhage as it did under PEG-induced hypovolaemia. Since the blood volume depletion is known to enhance the electrophysiological (Poulain \& Wakerley 1982), gene and secretory (Fenelon et al. 1993, Shoji et al. 1993) activities of magnocellular neurons, tGLP-1 is likely to influence each of these functions. For example, tGLP-1 was found to induce the release of some excitatory amino acid neurotransmitters in the hypothalamus (Calvo et al. 1995). Moreover, centrally injected tGLP-1 was shown to influence the gene activity in discrete brain structures (Van Dijk et al. 1996), some of them (e.g. the area postrema) being known to affect the hypothalamo-neurohypophysial system. It is also noteworthy that acute hypovolaemia is a stressful stimulus that leads to enhanced adrenocorticotrophin secretion (Lilly et al. 2000) and interoceptive stress has been shown to activate tGLP-1 neurons in the brain (Rinaman 1999). This may suggest a possible effect of tGLP-1 in the maintenance of body homeostasis under haemorrhage. Therefore, further experiments need to be aimed at establishing the possible involvement of endogenous tGLP-1 in the regulation of blood pressure and neurohypophysial hormone secretion under hypovolaemia.

Together, the present findings indicate that tGLP-1 may play a role in the adaptive mechanisms involved in the cardiovascular and hormonal response to both normo- and hypotensive hypovolaemia. Probably, tGLP-1 exerts its action through modulation of the neural activity including the function of vasopressinergic and oxytocinergic neurons.

\section{Acknowledgements}

The authors are very grateful to Professor Jan W Guzek for suggestions and critical reading of the manuscript. This work was supported by the Medical University of Lodz, contract No. 502-11-615.

\section{References}

Badoer E, McKinley MJ, Oldfield BJ \& McAllen RM 1992 Distribution of hypothalamic, medullary and lamina terminalis neurons expressing Fos after hemorrhage in conscious rats. Brain Research $\mathbf{5 8 2}$ 323-328.

Barragán JM, Eng J, Rodriguez R \& Blázquez E 1999 Neural contribution to the effect of glucagon-like peptide-1-(7-36) amide on arterial blood pressure in rats. American Journal of Physiology 277 E784-E791.

Bojanowska E \& Stempniak B 2000 Effects of centrally or systemically injected glucagon-like peptide-1 (7-36) amide on release of neurohypophysial hormones and blood pressure in the rat. Regulatory Peptides 91 75-81. 
Bojanowska E, Guzek JW \& Dabrowski R 1999 Luteinizing hormone-releasing hormone and function of the magnocellular vasopressinergic system. Neuropeptides 33 301-305.

Calvo JC, Gisolfi CV, Blázquez E \& Mora F 1995 Glucagon-like peptide-1(7-36)amide induces the release of aspartic acid and glutamine by the ventromedial hypothalamus of the conscious rat. Brain Research Bulletin 38 435-439.

Fenelon VS, Poulain DA \& Theodosis DT 1993 Oxytocin neuron activation and Fos expression: a quantitative immunocytochemical analysis of the effect of lactation, parturition, osmotic and cardiovascular stimulation. Neuroscience $\mathbf{5 3}$ 77-89.

Forsling ML, Kelestimur H \& Windle R 1991 The influence of reproductive status on vasopressin release in the rat. Journal of Endocrinology 130 387-393.

Göke R, Larsen PJ, Mikkelsen JD \& Sheikh SP 1995 Identification of specific binding sites for glucagon-like peptide- 1 on the posterior lobe of the pituitary. Neuroendocrinology 62 130-134.

Jin SLC, Han VKM, Simmons JG, Towle AC, Lauder JM \& Lund PK 1988 Distribution of glucagon-like peptide 1 (GLP-1), glucagon, and glicentin in the rat brain: an immunocytochemical study. Journal of Comparative Neurology 271 519-532.

Kadekaro M, Terrel ML, Liu H, Gestl S, Bui V \& Summy-Long JY 1998 Effects of L-NAME on cerebral metabolic, vasopressin, oxytocin, and blood pressure responses in hemorrhaged rats. American Journal of Physiology 274 R1070-R1077.

Kreymann B, Ghatei MA, Burnet P, Williams G, Kanse S, Diani AR \& Bloom SR 1989 Characterization of glucagon-like peptide-1-(7-36)amide in the hypothalamus. Brain Research $\mathbf{5 0 2}$ 325-331.

Larsen PJ, Tang-Christensen M \& Jessop DS 1997 Central administration of glucagon-like peptide-1 activates hypothalamic neuroendocrine neurons in the rat. Endocrinology 138 4445-4455.

Lewandowska A, Bojanowska E, Stempniak B \& Guzek JW 1995 Luliberin inhibits the release of oxytocin from hypothalamoneurohypophysial system in dehydrated but not in euhydrated or haemorrhaged rats. Endocrine Regulations 29 225-231.

Lilly MP, Jones RO, Putney DJ \& Carlson DE 2000 Post-surgical recovery and time-of-day mask potentiated responses of ACTH to repeated moderate hemorrhage in conscious rats. Journal of Endocrinology 167 205-217.

Merchenthaler I, Lane M \& Shughrue P 1999 Distribution of pre-pro-glucagon and glucagon-like peptide-1 receptor messenger RNAs in the rat central nervous system. Journal of Comparative Neurology 403 261-280.

Navarro M, de Fonseca FR, Alvarez E, Chowen JA, Zueco JA, Gomez R, Eng J \& Blázquez E 1996 Colocalization of glucagon-like peptide 1 (GLP-1) receptors, glucose transporter GLUT-2, and glucokinase mRNAs in rat hypothalamic cells: evidence for a role of GLP-1 receptor agonists as an inhibitory signal for food and water intake. Journal of Neurochemistry $6 \mathbf{7}$ 1982-1991.

Poulain DA \& Wakerley JB 1982 Electrophysiology of hypothalamic magnocellular neurones secreting oxytocin and vasopressin. Neuroscience 7 773-808.

Renaud LP 1996 CNS pathways mediating cardiovascular regulation of vasopressin. Clinical and Experimental Pharmacology and Physiology 23 157-160.

Rinaman L 1999 Interoceptive stress activates glucagon-like peptide-1 neurons that project to the hypothalamus. American Journal of Physiology 277 R582-R590.

Satoh F, Beak SA, Small CJ, Falzon M, Ghatei MA, Bloom SR \& Smith DM 2000 Characterization of human and rat glucagon-like peptide-1 receptors in the neurointermediate lobe: lack of coupling to either stimulation or inhibition of adenylyl cyclase. Endocrinology 141 1301-1309.

Schiltz JC, Hoffman GE, Stricker EM \& Sved AF 1997 Decreases in arterial pressure activate oxytocin neurons in conscious rats. American Journal of Physiology 273 R1474-R1483.

Share L 1996 Control of vasopressin release: an old but continuing story. News in Physiological Sciences 11 7-13.

Shoji M, Kimura T, Ota K, Inoue M, Sato K, Yamamoto T, Ohta M, Kawarabayasi Y \& Yoshinaga K 1993 Acute hypotensive hemorrhage stimulates vasopressin gene transcription in the rat brain. Annals of the New York Academy of Sciences 689 670-673.

Shughrue PJ, Lane M \& Merchenthaler I 1996 Glucagon-like peptide-1 receptor (GLP1-R) mRNA in the rat hypothalamus. Endocrinology 137 5159-5162.

Smith DW, Sibbald JR, Khanna S \& Day TA 1995 Rat vasopressin cell responses to stimulated hemorrhage: stimulus-dependent role for A1 noradrenergic neurons. American Journal of Physiology 268 R1336-R1342.

Stricker EM \& Verbalis JG 1986 Interaction of osmotic and volume stimuli in regulation of neurohypophyseal secretion. American Journal of Physiology 250 R267-R275.

Szczepańska-Sadowska E 1996 Interaction of vasopressin and angiotensin II in control of blood pressure and thirst. Regulatory Peptides 66 65-71.

Thrasher TN \& Keil LC 2000 Systolic pressure predicts plasma vasopressin responses to hemorrhage and vena caval constriction in dogs. American Journal of Physiology 279 R1035-R1042.

Ueta Y, Levy A, Lightman SL, Hara Y, Serino R, Nomura M, Shibuya I, Hattori K \& Yamashita H 1998 Hypovolemia upregulates the expression of neuronal nitric oxide synthase gene in the paraventricular and supraoptic nuclei of rats. Brain Research $\mathbf{7 9 0}$ $25-32$.

Ullman J 2000 Influence of neurohumoral blockade on heart rate and blood pressure responses to haemorrhage in isoflurane anaesthetized rats. Acta Physiologica Scandinavica 169 189-194.

Van Dijk G, Thiele TE, Donahey JCK, Campfield LA, Smith FJ, Burn P, Bernstein IL, Woods SC \& Seeley RJ 1996 Central infusions of leptin and GLP-1-(7-36) amide differentially stimulate c-FLI in the rat brain. American Journal of Physiology 271 R1096-R1100.

Yamaguchi K, Hama H \& Watanabe K 1998 Possible participation of prostaglandins generated in the anteroventral third ventricular region in the hypovolemia-induced vasopressin secretion of conscious rats. European Journal of Endocrinology 138 206-215.

Yokoi H, Arima H, Murase T, Kondo K, Iwasaki Y \& Oiso Y 1996 Intracerebroventricular injection of adrenomedullin inhibits vasopressin release in conscious rats. Neuroscience Letters 216 65-67.

Zueco JA, Esquifino AI, Chowen JA, Alvarez E, Castrillón PO \& Blázquez E 1999 Coexpression of glucagon-like peptide-1 (GLP-1) receptor, vasopressin, and oxytocin mRNAs in neurons of the rat hypothalamic supraoptic and paraventricular nuclei: effect of GLP-1 (7-36) amide on vasopressin and oxytocin release. Journal of Neurochemistry 72 10-16.

Received 26 October 2001 Accepted 31 October 2001 